\title{
Experimental and numerical investigation of capillary flow in SU8 and PDMS microchannels with integrated pillars
}

Saha, AA., Mitra, SK., Tweedie, M., Roy, SS., \& McLaughlin, JAD. (2009). Experimental and numerical investigation of capillary flow in SU8 and PDMS microchannels with integrated pillars. Microfluidics and Nanofluidics, 17(1), 15. https://doi.org/10.1007/s10404-008-0395-0

Link to publication record in Ulster University Research Portal

\section{Published in:}

Microfluidics and Nanofluidics

Publication Status:

Published (in print/issue): 01/01/2009

DOI:

10.1007/s10404-008-0395-0

\section{Document Version}

Publisher's PDF, also known as Version of record

\section{General rights}

Copyright for the publications made accessible via Ulster University's Research Portal is retained by the author(s) and / or other copyright owners and it is a condition of accessing these publications that users recognise and abide by the legal requirements associated with these rights.

\section{Take down policy}

The Research Portal is Ulster University's institutional repository that provides access to Ulster's research outputs. Every effort has been made to ensure that content in the Research Portal does not infringe any person's rights, or applicable UK laws. If you discover content in the Research Portal that you believe breaches copyright or violates any law, please contact pure-support@ulster.ac.uk. 


\title{
Experimental and numerical investigation of capillary flow in SU8 and PDMS microchannels with integrated pillars
}

\author{
Auro Ashish Saha · Sushanta K. Mitra • \\ Mark Tweedie $\cdot$ Susanta Roy $\cdot$ Jim McLaughlin
}

Received: 2 October 2008/Accepted: 9 December 2008/Published online: 8 January 2009

(C) Springer-Verlag 2008

\begin{abstract}
Microfluidic channels with integrated pillars are fabricated on SU8 and PDMS substrates to understand the capillary flow. Microscope in conjunction with highspeed camera is used to capture the meniscus front movement through these channels for ethanol and isopropyl alcohol, respectively. In parallel, numerical simulations are conducted, using volume of fluid method, to predict the capillary flow through the microchannels with different pillar diameter to height ratio, ranging from 2.19 to 8.75 and pillar diameter to pitch ratio, ranging from 1.44 to 2.6. The pillar size (diameter, pitch and height) and the physical properties of the fluid (surface tension and viscosity) are found to have significant influence on the capillary phenomena in the microchannel. The meniscus displacement is non-uniform due to the presence of pillars and the nonuniformity in meniscus displacement is observed to increase with decrease in pitch to diameter ratio. The surface area to volume ratio is observed to play major roles in the velocity of the capillary meniscus of the devices. The filling speed is observed to change more dramatically under different pillar heights upto $120 \mu \mathrm{m}$ and the change is slow with further increase in the pillar height. The details pertaining to the fluid distribution (meniscus front shapes) are
\end{abstract}

A. A. Saha

Department of Mechanical Engineering,

Indian Institute of Technology Bombay, Mumbai, India

S. K. Mitra $(\bowtie)$

Department of Mechanical Engineering, University of Alberta,

Edmonton, Canada

e-mail: sushanta.mitra@ualberta.ca

M. Tweedie $\cdot$ S. Roy $\cdot$ J. McLaughlin

Nanotechnology and Integrated BioEngineering Centre,

University of Ulster, Jordanstown, Northern Ireland obtained from the numerical results as well as from experiments. Numerical predictions for meniscus front shapes agree well with the experimental observations for both SU8 and PDMS microchannels. It is observed that the filling time obtained experimentally matches very well with the simulated filling time. The presence of pillars creates uniform meniscus front in the microchannel for both ethanol and isopropyl alcohol. Generalized plots in terms of dimensionless variables are also presented to predict the performance parameters for the design of these microfluidic devices. The flow is observed to have a very low Capillary number, which signifies the relative importance of surface tension to viscous effects in the present study.

Keywords Capillary flow $\cdot$ Microchannel $\cdot$ Numerical . Three-dimensional

\section{Introduction}

Microchannels are used for the transport of minute amount of liquids for cell-sorting and cellular assays, protein crystallization, immunoassays, DNA analysis, and medical applications (Zimmermann et al. 2005). Precise liquid volume control and guiding fluid flow to the desired outlet reservoir are two important operations in Lab-on-a-Chip design. Passive systems typically rely upon the balance of surface tension and fluid pressure forces to perform their function. In particular, it is possible to exploit surface tension forces to create passive microfluidic valves and metering systems. When capillary action is used for microfluidics, the wetting property of microchannels has a significant effect on the liquid behavior. It is very wellknown fact that, a hydrophilic surface assists fluid motion 
whereas, a hydrophobic surface retards fluid motion inside microchannels. Pillar structures are often used in microchannels to increase the surface/volume ratio and increase the capillary flow. The pillar structures have also found to increase the yield of cell capture from blood (Thorslund et al. 2008). An important consideration in the design of the inlet/exit ports of microfluidic devices is to use of micropillars. It is a common practice to include arrays of micromachined posts that serve to modify fluid flow and act as sites for heterogeneous catalysis (Losey et al. 2002).

Son et al. (2006) used a PDMS structure with a pillar array $(200 \mu \mathrm{m}$ in diameter, $250 \mu \mathrm{m}$ in distance between pillars, and $1 \mathrm{~mm}$ in height) for filtration of beads. They used disposable bio-chip for immobilizing hemoglobin Alc (HbAlc) and to measure its concentration. Nissila et al. (2007) developed a lidless micropillar array electrospray ionization chip for analysis of drugs and biomolecules. The chip made of silicon, consisted of an array of micropillars in an open-channel and an electrospray emitter tip for ionization. The micropillar array provided the necessary liquid transfer in the chip by capillary force.

Over the last few years, attempts have been made by number of researchers to simulate such passive transport in microchannels. Tseng et al. (2002) applied the volume of fluid (VOF) interface tracking technique to understand the reservoir filling process, and investigated factors such as the contact angle and reservoir shape on the filling process. They verified their simulation using a microscale particle image velocimetry ( $\mu$-PIV). They identified hydrophilic/ hydrophobic nature of the wall as the key issue for liquid filling into the micro-reservoir for the channel/reservoir of $1 \mathrm{~mm}$ in size. It was further demonstrated experimentally that, the complete filling becomes difficult for their devices with feature size around $100 \mu \mathrm{m}$, even with hydrophilic wall.

Quinte et al. (2001) present the validation of the commercial simulation tools FLOW-3D and CFX4 with regard to capillary driven flows in two-dimensional rectangular channels. The computational time ranged between a few minutes to hours for the elementary microchannel configurations considered in their study. They verified their simulation with analytical solution and experimental data. The measurement data showed lower values of average filling velocity than those obtained through analysis and simulations. They also proposed capillary driven flow in a three-dimensional microchannel with integrated columns for a medical test strip. However, simulation for the threedimensional microchannel with integrated columns was not carried out in their work.

Microchannels with integrated pillared structures will form an integral component in the development of biomedical chips (Mery et al. 2008). Micropillars array also help to deliver fluid from the reservoir to microfluidic channels (Jokinen and Franssila 2008). Capillary action, which is purely governed by the surface tension forces (Probstein 1994), is used for the transport of the fluid into these devices. The wall surfaces of the devices exhibit different characteristics based on the substrate material used during their fabrication. There is a need to understand the flow behavior in these devices in order to accurately design the high performance chips. Due to the complexity of the geometry, it is often difficult to formulate analytical solutions. Analytical models have been proposed for modeling of underfill process in the flip-chip assembly (Wan et al. 2005, 2007; Lin et al. 2007; Lin 2004). However, the details pertaining to the fluid distribution (meniscus front shapes) cannot be obtained from the theoretical analysis based on these analytical models. In the numerical study for flip-chip underfill flow (Wan et al. 2005), the calculated filling time matched well with the measured results, whereas, the flow front shape did not match well with the measured results. The front shapes, which is a measure of, how well the fluid gets distributed, is an important parameter to ensure reliability and performance of microfluidic devices. Hence it becomes necessary to rely on numerical techniques to predict the flow phenomena in such cases. The general features of free surface flow in microchannels requires enormous computational time based on the complexity of geometry of the flow under study. To the best of the authors knowledge, limited work has been conducted to study the capillary flow in microchannel with integrated pillars.

We present a three-dimensional simulation of free surface phenomena in a microfluidic channel containing $10 \times 15$ array of $350 \mu \mathrm{m}$ circular cross-section pillars by VOF technique. The VOF technique, is the most commonly used technique for the simulation of free surfaces within commercial and academic CFD packages (Jimack 2004). Microfluidic imaging is used to experimentally visualize the interface movement on the fabricated microchannels using SU8 and PDMS substrates. This helps in understanding how free surface is modified by the presence of pillars for performing microfluidic analysis. A parametric study is also performed to understand the effects of surface tension and viscosity of the working fluid on the geometry of the integrated pillars.

\section{Microchannel fabrication and characterization}

The microfluidic devices fabricated from polymer materials such as PDMS have simpler manufacturing procedures and are comparatively cheaper and particularly used for disposable microfluidic devices. There has also been a growing interest in using organic-based, negative tone photoresists, such as the epoxy-derived material, SU8 in 
biofluidics applications. Hence, microchannels made of SU8 and PDMS consisting of arrays of circular pillar are considered in this study. However, PDMS and SU8 are low surface energy materials and are innately hydrophobic, and do not adhere well to other materials brought in contact. This necessitates their surface modification/treatment to render them adhesionable. The modified/treated surface recovers its hydrophobicity very shortly (Kim et al. 2004). In the present study, the surface of the PDMS/SU8 have not been modified. The SU8 and PDMS channels are sealed with glass slides which is hydrophilic in nature and thus will assist fluid motion under passive surface tension forces.

\subsection{SU8 substrate}

The SU8 channel is fabricated from glass microscope slides patterned with SU8 photoresist using an SF 100 maskless lithography system (Kern et al. 2007). Subsequently, microscope slide have been adhered with cyanoacrylate adhesive for sealing the device. The pillars are $350 \mu \mathrm{m}$ diameter and $120 \mu \mathrm{m}$ height (confirmed with stylus profilometer), with adjacent pillars being separated by $300 \mu \mathrm{m}$. Figure 1a shows the optical micrograph of the device after sealing.

\subsection{PDMS substrate}

The microchannel was fabricated by PDMS (Sylgard 184, Dow Corning, Co.) molding on a master pattern made using SU8 photoresist on silicon wafer (McDonald and Whitesides 2002). The cured pattern was peeled from the master and sealed using microscope glass slide to obtain a reversible sealing. The wafer containing the pattern is coated with a thin layer of silane to make it easier to peel the solidified PDMS from the wafer. Figure $1 b$ shows the optical micrograph of the device fabricated on PDMS substrate. It is observed that, the dimensional accuracy of PDMS pillars is not as good as compared with SU8 pillars. It is to be noted that, the PDMS fabrication technique is cheap and easy but a considerable drawback is that the reproduction of the stamp pattern is not precise due to the wetting properties of liquid and stamp (Seemann et al. 2004). Also, there may be dimensional inaccuracies related with the master mold used for PDMS fabrication. The pillar dimensions are $330 \mu \mathrm{m}$ diameter with inter separation distance of $320 \mu \mathrm{m}$, between the pillars. The pillar height is $40 \mu \mathrm{m}$, when measured with stylus profilometer.

The analysis of the roughness of the microchannel bottom surface is performed by the surface profilometer (AMBIOS XP2). The average channel wall roughness in the fabricated microchannel is $\sim 3.32 / 8.54 \mathrm{~nm}$ for glass/ SU8 surface and $\sim 0.46 \mu \mathrm{m}$ for PDMS surface, respectively. The effects of roughness is often modelled by the Wenzel equation (Jensen 2002), when the contact angles less than $90^{\circ}$ are decreased and angles greater than $90^{\circ}$ are increased by roughness. As the roughness values are very small compared with the channel size (40 and $120 \mu \mathrm{m})$ considered here, the roughness effect is neglected in this present study. Moreover, the fluids under consideration (isopropyl alcohol and ethanol) have high wetting characteristics and contact angles considered here are less than $90^{\circ}$, hence surface roughness will have minimal effects in the present analysis. Implications of hydrophobic interactions such as apparent slip mechanisms in narrow fluidic
Fig. 1 Optical micrograph of microchannel showing the pillars in top view. a SU8 microchannel, b PDMS microchannel
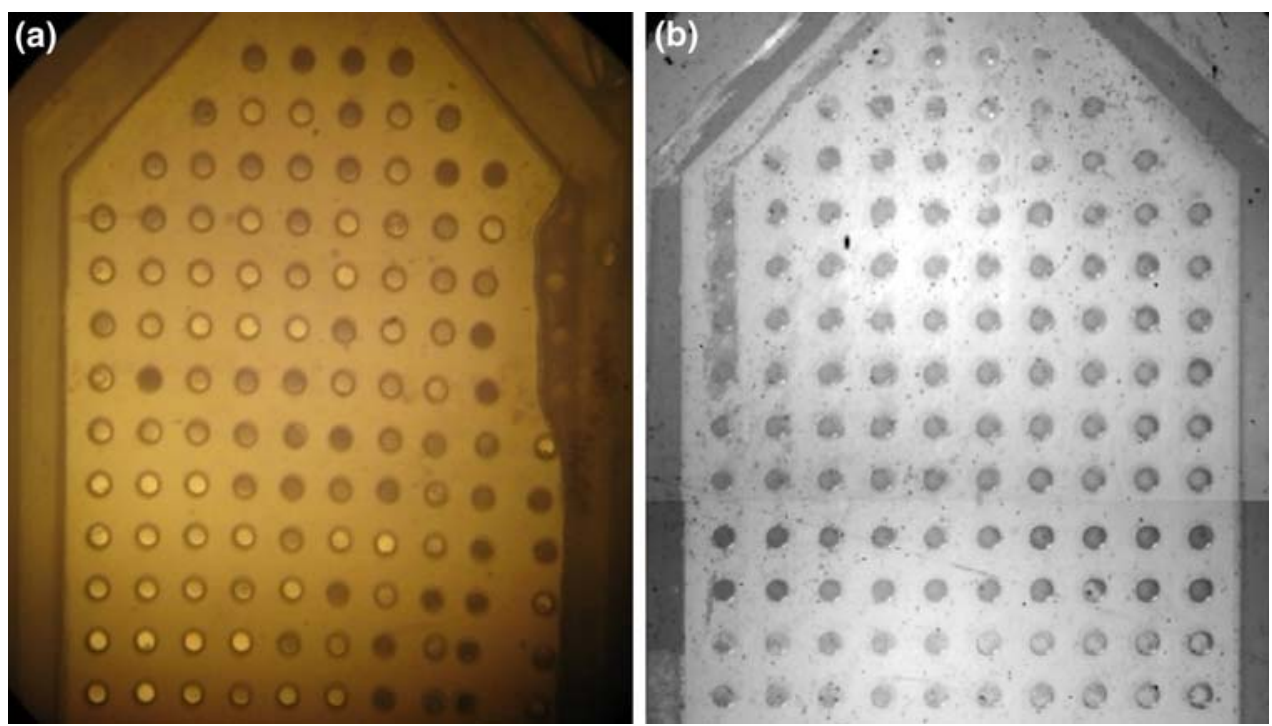
confinements induced by surface roughness-hydrophobicity coupling have been discussed in the recent literature (Chakraborty 2007a, b; Chakraborty et al. 2007; Chakraborty and Dinkar 2008).

\section{Numerical simulation}

\subsection{Geometry of microchannel}

The geometry of the microchannel model is shown in Fig. 2. The channel considered here is a three-dimensional channel with array of circular pillars of $350 \mu \mathrm{m}$ crosssection. The substrate materials for the microchannel under consideration are Polydimethylsiloxane (PDMS) and SU8. The pillars made of SU8/PDMS are arranged in line and separated by a distance of $300 \mu \mathrm{m}$. The pillar height is $120 \mu \mathrm{m}$ for SU8 and $40 \mu \mathrm{m}$ for PDMS microchannel, respectively.

\subsection{Governing equations}

The transient, three-dimensional numerical simulations of the capillary flow in the microchannel with integrated pillars are performed using VOF method (Hirt and Nichols 1981). The system consists of two incompressible and immiscible fluids represented as liquid and gas phases. Surface tension effects are incorporated in the VOF method. The fluids under consideration are ethanol and isopropyl alcohol as liquid and air as gas. In this method, we solve momentum equation and continuity equation. The flow is considered to be laminar, incompressible, Newtonian and isothermal with velocity field $\mathbf{V}$ governed by the Navier-Stokes and continuity equations, which can be written as:

$\nabla \cdot \mathbf{V}=0$

$\frac{\partial \rho \mathbf{V}}{\partial t}+\nabla \cdot(\rho \mathbf{V V})=-\nabla P+\rho \mathbf{g}+\nabla \cdot\left(\mu\left(\nabla \mathbf{V}+\nabla^{T} \mathbf{V}\right)\right)+\mathbf{F}_{s}$

where $\mathbf{V}$ is the velocity of the mixture, $P$ the pressure, $t$ the time, $\mathbf{F}_{s}$ the volumetric force at the interface resulting from surface tension, and $\rho, \mu$ are the density and dynamic viscosity, respectively. In Eq. 2, the accumulation and convective momentum terms in every control volume (cell) balance the pressure force, gravity force, shear force, and additional surface tension force $\mathbf{F}_{s}$.

The physical properties of each fluid are calculated as weighted averages based on the volume fraction of the individual fluid in a single cell. The fluid volume in a cell is computed as $F_{\text {vol }}=F V_{\text {cell }}$, where $V_{\text {cell }}$ is the volume of a computational cell and $F$ is the liquid volume fraction in a

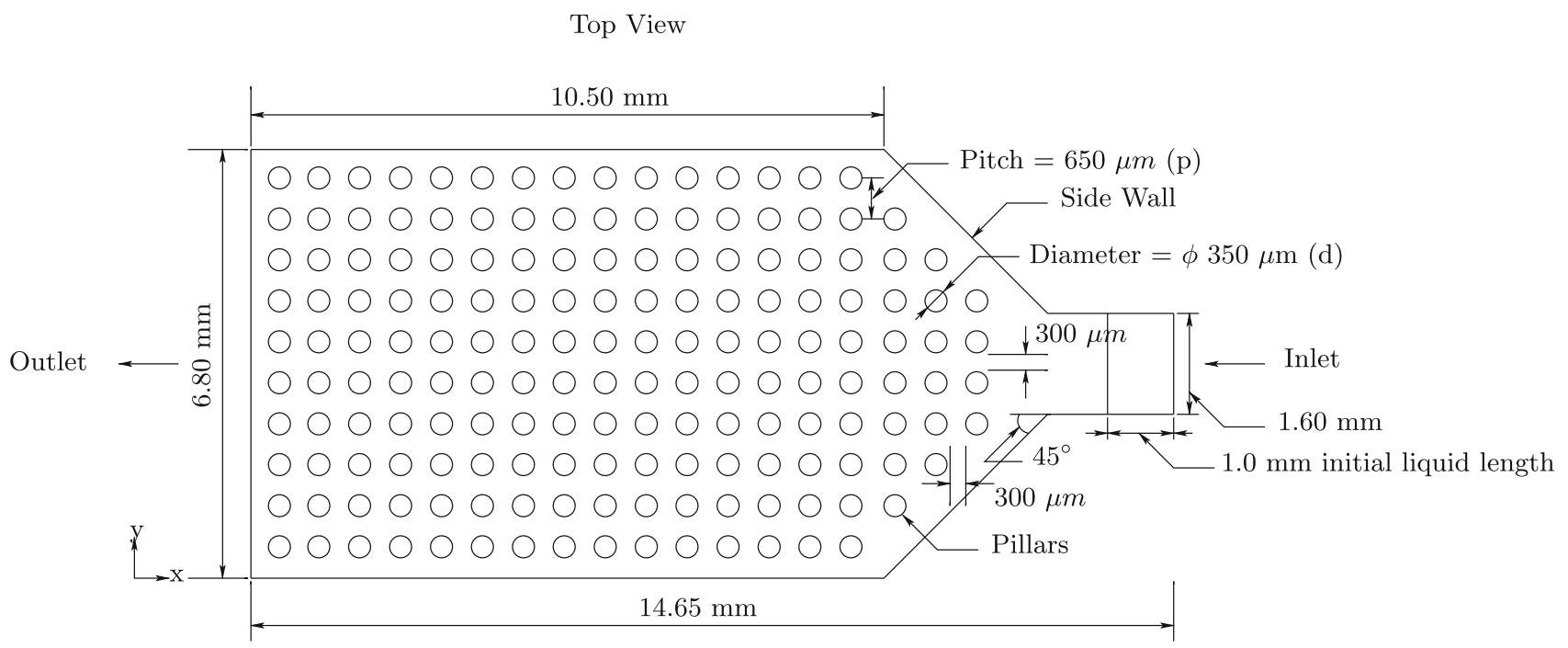

Front View

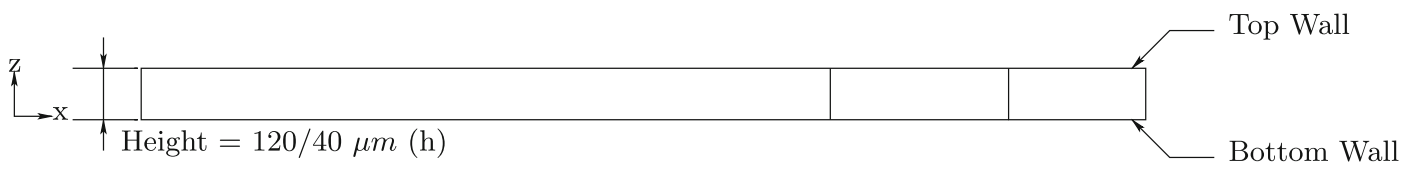

Fig. 2 Geometry of the microchannel 
cell. The value of $F$ in a cell should range between 1 and 0 . Here, $F=1$ represents a cell which is completely filled with liquid, $F=0$ represents a cell which is completely filled with gas and $0<F<1$ represents the liquid/gas interface.

The liquid volume fraction distribution can be determined by solving a separate passive transport equation, given as:

$\frac{\partial F}{\partial t}+\mathbf{V} \cdot \nabla F=0$

where,

$F=\frac{\text { cell volume occupied by liquid }}{\text { total volume of the control cell }}$

The mixture's physical properties are derived from that of the two phases through the volume fraction function. In particular, the average value of $\rho$ and $\mu$ in a computational cell can be computed from the value of $F$ in accordance with:

$\rho=F \rho_{2}+(1-F) \rho_{1}$

$\mu=F \mu_{2}+(1-F) \mu_{1}$

where the subscripts 1 and 2 represent the gas and the liquid phases, respectively.

The surface tension model follows the continuum surface force (CSF) model proposed by Brackbill et al. (1992). The surface tension in Eq. 2 according to the CSF model is computed as:

$\mathbf{F}_{s}=\sigma \kappa \nabla F$

The surface tension is taken to be constant along the surface and only the forces normal to the interface are considered. According to the CSF model, the surface curvature $\kappa$ is computed from local gradients in the surface normal to the interface, which is given as:

$\kappa=\frac{1}{|\mathbf{n}|}\left(\left(\frac{\mathbf{n}}{|\mathbf{n}|} \cdot \nabla\right)|\mathbf{n}|-\nabla \cdot \mathbf{n}\right)$

where $\mathbf{n}=\nabla F$ is the normal vector. Wall adhesion is included in the model through the contact angle:

$\hat{\mathbf{n}}=\hat{\mathbf{n}}_{w} \cos \theta+\hat{\mathbf{t}}_{w} \sin \theta$

where $\hat{\mathbf{n}}$ is the unit vector normal to the surface, $\hat{\mathbf{n}}=\frac{\mathbf{n}}{|\mathbf{n}|}, \hat{\mathbf{n}}_{w}$ and $\hat{\mathbf{t}}_{w}$ represents the unit vector normal and tangent to the wall, respectively.

Equations 1-9 are solved iteratively to obtain the liquid volume fraction and the velocity field solution under appropriate initial and boundary conditions. The effect of gravity is not taken into account in the present study, as the Bond number (Bo), $\rho g h^{2} / \sigma$ is much less than unity, where $h$ is the height of the microchannel, $g$ is the acceleration due to gravity, $\rho$ and $\sigma$ are the fluid density and surface tension coefficient, respectively.

\subsection{Initial and boundary conditions}

Initially, at time $t=0$, the liquid meniscus position in the channel is set as $1.00 \mathrm{~mm}$ from the inlet. No slip boundary condition is imposed on all the walls and the boundary conditions for the surface affinity are described by the contact angles. The contact angle value of zero is specified on all the walls of the channel as shown in Fig. 2, as the test liquids ethanol and isopropyl alcohol offer very high wetting properties for glass, PDMS and SU8 surfaces. It may also be noted that, the test liquids being highly volatile, the vapors increase the saturation of the solid surface, thus exhibiting a behavior of a prewetted capillary (Barraza et al. 2002; Xiao et al. 2006). This is analogous to treating a wall having a fully wet condition with a contact angle of $0^{\circ}$, where the actual energetics of wetting the wall do not matter (Jokinen and Franssila 2008). Passive capillary filling process is considered by specifying a constant pressure (atmospheric) at channel inlet and outlet. A liquid volume fraction value of unity and zero is specified at channel inlet and outlet, respectively.

\subsection{Solution technique}

Open source based CFD analysis code OpenFOAM 1.5 (Open Field Operation and Manipulation) which is written by OpenCFD Ltd is used for the simulations here. The solution technique follows the finite volume numerics to solve systems of partial differential equations ascribed on any 3D unstructured mesh of polyhedral cells (OpenCFD 2008). The solver implements the VOF two-phase algorithm for the computations which enables the capturing of sharp fluid/fluid interfaces (Ubbink and Issa 1999; Rusche 2002). As a transient solution is desired for the present capillary driven flow, the selection of the time step has to be based such that the stability of the numerical simulation is ensured. Therefore, a target Courant-Friedrich's-Lewy (CFL $=|v|$ $\delta t / h$, where $v$ is the interface velocity, $h$ is the local cell dimension and $t$ is time) number of 0.1 is applied for numerical stability of the simulation. This would allow the interface to cross $10 \%$ of the width of a grid cell during each time step in a VOF computation. Pressure Implicit with Splitting of Operators (PISO) algorithm is adopted for pressure-velocity coupling and pressure correction. The necessary compression of the interface is achieved by introducing an additional artificial compression term into the VOF equation $\left(\nabla \cdot(F(1-F)) V_{r}\right)$, where $V_{r}$ is a velocity field suitable to compress the interface. This artificial term is active only in the interface region due to the term $F(1-F)$. It also helps in keeping the interface without getting separated, particularly for diverging flows. Initial time step of $1.0 \mathrm{E}-09 \mathrm{~s}$ is selected and the time step is allowed to automatically adjust based on the CFL number and interface 
velocity which ensures the stability in the solver. A convergence criteria of 0.0001 is specified to control the iterative solution process. In the numerical simulation, the symmetry boundary condition about the center plane is used because of the symmetric flow along the width and thickness of the microchannel, and thus quarter of the geometry is only computed. The isocontour of $F=0.5$ is usually applied to identify the interfacial position for computation and visualization purposes. The computations are performed within a clustered parallel environment based on open message passing interface (OpenMPI) library and the mesh has been decomposed using automatic load-balanced decomposition method (Metis). The physical properties of ethanol and isopropyl alcohol used for simulations are provided in Table 1.

\subsection{Parallel performance}

The numerical study of free surface phenomena requires enormous computational resources. A parallel performance test was done with the three-dimensional microchannel geometry, as shown in Fig. 2, for 266,656 cells. The decomposition of the domain in 2, 3, 4, 6 and 8 subdomains were made using the automatic load-balanced decomposition (Metis). A Linux cluster was setup consisting of 4 node Dual Core AMD Opteron processors with 2 GB RAM, i.e. 2 cores (CPUs) per node and a total of 8 cores (CPUs). The Fedora Linux operating system was used and each node had the separate installation of the solver executables with a common NFS working directory shared among the nodes. The simulation starts at $0 \mathrm{~s}$ and is allowed to run for $0.001 \mathrm{~s}$. The wall clock times measured for different CPU configurations is presented in Table 2. It is observed that the execution time for the simulation decreases with the increase of CPU usage. The parallel speed up is linear upto to 4 CPUs and any further increase in CPU usage, the parallel efficiency is found to be significantly reduced. It is further observed that the speed up obtained is higher when distributing the processes on different nodes than using the multi-core CPUs in each node. The memory sharing in the nodes becomes a bottleneck when computations are carried using the multi-core CPUs on each node.

Table 1 Fluid properties used in simulation

\begin{tabular}{llll}
\hline Physical property & Ethanol & Isopropyl alcohol & Air \\
\hline Density $\left(\mathrm{kg} / \mathrm{m}^{3}\right)$ & 791 & 785 & 1.1614 \\
Viscosity $(\mathrm{mPa} \mathrm{s})$ & 1.2 & 2.43 & 0.0185 \\
Surface tension $(\mathrm{N} / \mathrm{m})$ & 0.0214 & 0.0228 & - \\
\hline
\end{tabular}

Table 2 Parallel performance

\begin{tabular}{llll}
\hline No. of CPU & No. of nodes & Execution time (s) & Speed up \\
\hline 1 & 1 & 4,526 & 1.00 \\
2 & 1 & 3,010 & 1.50 \\
2 & 2 & 2,308 & 1.96 \\
3 & 3 & 1,637 & 2.76 \\
4 & 2 & 1,546 & 2.93 \\
4 & 4 & 1,307 & 3.46 \\
6 & 3 & 1,157 & 3.91 \\
8 & 4 & 1,109 & 4.08 \\
\hline
\end{tabular}

\section{Experiments}

\subsection{Flow visualization}

Interfacial flow visualization is essential for tracking the transient flow in the microfluidic devices. The details of microfluidic imaging experimental setup is described in this section. We have used two different imaging experimental setup for measuring the meniscus profile and velocity of the meniscus front in the microchannel. The two experimental setup has been selected, due to the advantages each of them offer for the specific measurements in SU8 and PDMS microchannels. High resolution images of the transient evolution of the meniscus profile for SU8 microchannel is obtained using a inverted microscope (Nikon Eclipse TE2000-S) fitted with the designated objective (i.e. $1 \times, 2 \times, 4 \times, 10 \times, 20 \times, 40 \times, 60 \times$ ). The microscope is attached to a camera port to mount CCD camera (15 fps) for acquiring images within a predetermined time sequence. Halogen lamp is used for illumination. The cooled CCD camera has a resolution of $2,048 \times 2,048$ pixels, 16 bit per pixel for recording the images. The visualization is carried at $1 \times$ magnification, and only partial field of view is available for the microchannel. However, clear images could not be obtained with PDMS microchannel as the thick PDMS substrate exposed to the objective lens in the inverted microscope could not provide proper image focus. Imaging of the transient evolution of the meniscus front for PDMS microchannel is done using the stereo microscope fitted with the designated objective (i.e. $1 \times, 2 \times$ ). The microscope allows imaging of test samples at lower power magnification and provides a higher field of view with adequate working distance between the microscope objective and test sample. A digital camera is attached to the microscope eye piece for acquiring high frame rate video images $(60 \mathrm{fps}$ at $320 \times 240$ pixels resolution). The visualization is carried at $1 \times$ magnification to get the full field of view of the microchannel. 


\section{Results and discussion}

\subsection{Simulation results}

\subsubsection{Validation of numerical solution with analytical solution for a two-dimensional and three- dimensional rectangular microchannel}

Validation of the numerical results with analytical solution has been carried out for a rectangular microchannel $40 \mu \mathrm{m}$ in height, 7,000 $\mu \mathrm{m}$ length and $3000 \mu \mathrm{m}$ wide with black ink (Quinte et al. 2001) as working fluid ( $\rho=1032.2 \mathrm{~kg} /$ $\mathrm{m}^{3}, \sigma=0.072 \mathrm{~N} / \mathrm{m}$ and $\mu=0.00137 \mathrm{~Pa} \mathrm{~s}$ ) with contact angle $\theta=36^{\circ}$. The time evolution of meniscus displacement is considered as the parameter for validating the numerical results.

The analytical solution is based on a reduced-order model (Zeng 2007) and is derived here for clarity. For the channel height of $120 \mu \mathrm{m}$ selected in this study, the Capillary number $(\mathrm{Ca}), \mu U / \sigma$, is much less than unity and hence, the effects of dynamic contact angle are not taken into account in the present study (Huang et al. 2006; Bayramli and Powell 1989). A more elaborate approach for a capillary flow in two-dimensional channel has been considered including the effects of added or virtual mass, meniscus traction regime and dynamical evolution of the contact angle in (Chakraborty 2005, 2007c; Chakraborty and Mittal 2007; Chakraborty and Tsuchiya 2008). A reduced order model (Zeng 2007) which accounts for inertial forces is considered for validating the numerical results with analytical solution for a rectangular microchannel. The momentum conservation in a twodimensional microchannel can be expressed in terms of a balance between the surface tension force, pressure overhead and wall viscous force. At time $t$, if $L$ is the distance travelled by the liquid meniscus and $u_{\text {avg }}$ is the average fluid velocity, then the momentum balance can be written as,

$\frac{\mathrm{d}}{\mathrm{d} t}\left(\rho h L u_{\mathrm{avg}}\right)=2 \sigma \cos \theta+\Delta P h-\frac{12 \mu L}{h} u_{\mathrm{avg}}$

For a passive capillary filling process, $\Delta P=0$. Noting $u_{\mathrm{avg}}=\mathrm{d} L / \mathrm{d} t$, the equation above can be re-written as

$\frac{\mathrm{d}^{2}}{\mathrm{~d} t^{2}} L^{2}+B \frac{\mathrm{d}}{\mathrm{d} t} L^{2}=A$

where,

$A=\frac{4 \sigma \cos \theta+2 \Delta \mathrm{Ph}}{\rho h}, \quad B=\frac{12 \mu}{\rho h^{2}}$

Considering the initial liquid meniscus position in the channel as $L_{0}$ and with zero velocity, the transient solution of the capillary filling problem is given as:
$L=\left(\frac{A}{B^{2}} \exp (-B t)+\frac{A t}{B}+\left(L_{0}^{2}-\frac{A}{B^{2}}\right)\right)^{\frac{1}{2}}$

$u_{\mathrm{avg}}=\frac{A(1-\exp (-B t))}{2 B L}$

Figure 3 shows the comparison of meniscus displacement obtained numerically with the analytical solution based on the reduced-order model (Zeng 2007). Good agreement between numerical and analytical results is observed which provides confidence on the current model formulation. The threedimensional results also show good match with analytical solution, indicating that a two-dimensional solution would be adequate for this rectangular microchannel geometry due to the small height of channel in comparison with width $(w \gg h)$ (Chakraborty 2005).

The numerical solution of free surface flow is very much dependent on the quality of the grid (Sethian and Smereka 2003). A $50 \times 50 \times 10 \mu \mathrm{m}^{3}$ cell size is used for generating the grid in the microchannel geometry for all the cases considered in this study. The grid independence has been conducted by doubling the grid nodes but less than $5 \%$ difference in the flow fields were obtained.

The comparison of the simulated filling time of the capillary meniscus is shown in Fig. 4 for ethanol and isopropyl alcohol. It is observed that the capillary driven flow produces a non-linear displacement of the meniscus. It is found that the filling time for ethanol is lower compared to isopropyl alcohol for both SU8 and PDMS channels. This can be attributed to the lower viscosity of ethanol as compared to isopropyl alcohol.

Figure 5 shows the time evolution of the meniscus centerline velocity for PDMS microchannel with isopropyl alcohol. The velocity of the advancing liquid front at $\mathrm{x}$-position is obtained by the derivative of the liquid front

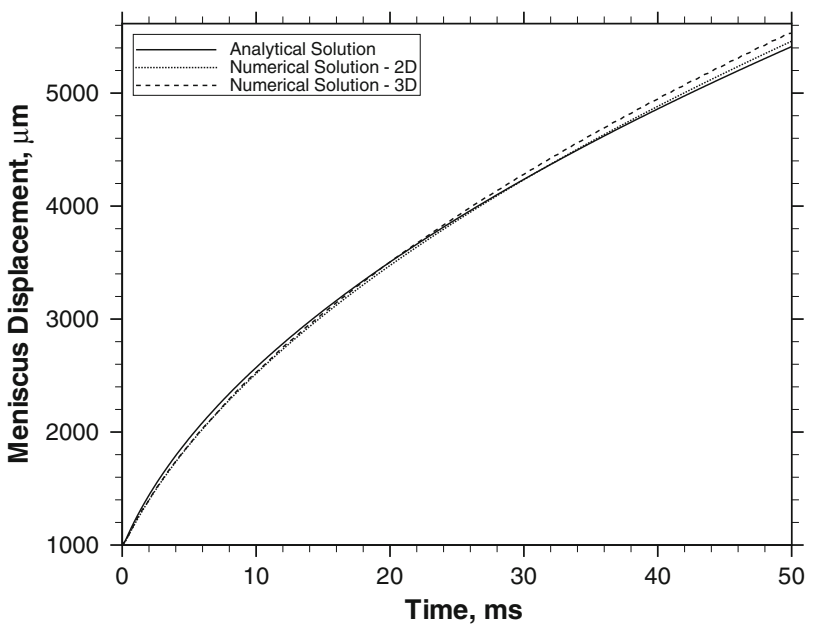

Fig. 3 Comparison of two-dimension and three-dimension simulated transient meniscus centerline displacement with analytical solution 


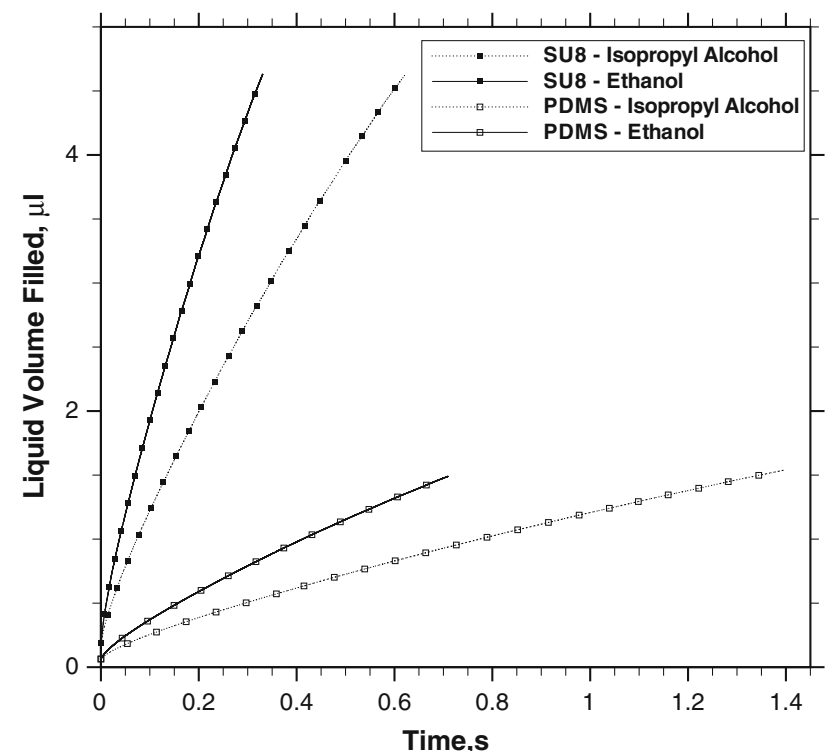

Fig. 4 Numerical comparison of liquid volume filling time of capillary meniscus

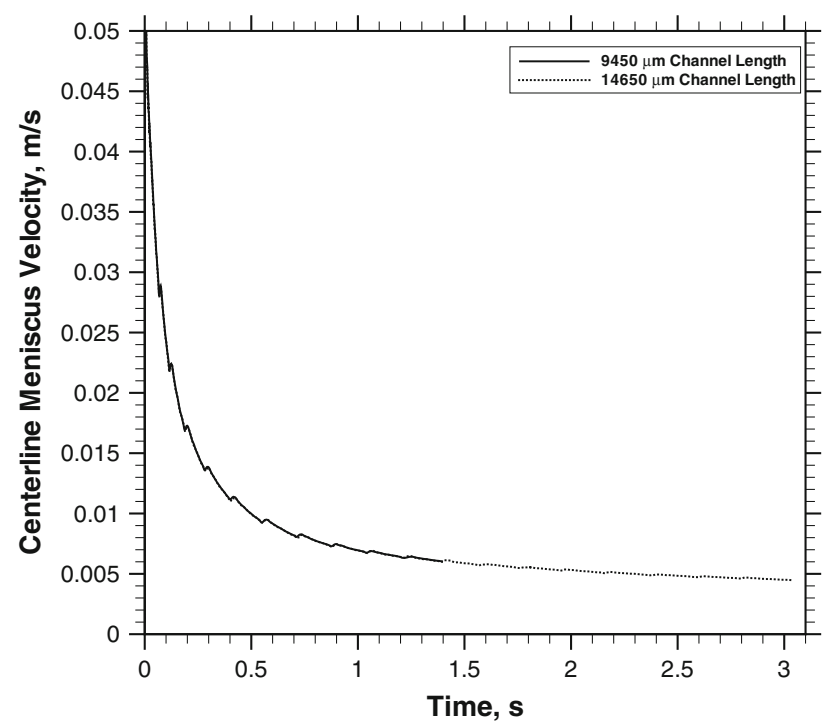

Fig. 5 Transient meniscus centerline velocity for PDMS microchannel with isopropyl alcohol. Dotted line original channel length, solid line reduced channel length

position (Chen et al. 2008) with time at time $=t$. The centerline velocity decreases with time. This being a nonsteady process, the interface is in nonequilibrium state relaxing to a configuration of minimum free energy and hence, the velocity is observed to be a decreasing function of time (Barraza et al. 2002). As the fluid enters the microchannel the viscous forces dominate at the entrance region and there is sharp decrease in velocity. When the flow becomes fully developed, the viscous drag is over come by capillary force. The continuous decrease in velocity is due to the increase of fluid accumulation in the microchannel which has to flow with the meniscus front. It is also observed that the simulation results remain unchanged if the channel length is reduced by half of the original length. Similar velocity profiles are obtained when considering the channel length of 14,650 and $9450 \mu \mathrm{m}$. Hence, to save computational time, simulations are carried out for the channel length of $9,450 \mu \mathrm{m}$ for all the cases considered in this study.

Figure 6 shows the filling time of capillary meniscus for different pillar diameter to height ratio $(d / h)$. The diameter is fixed at $350 \mu \mathrm{m}$ while the height of the pillar considered are $160,120,80,60$ and $40 \mu \mathrm{m}$ respectively. The filling speed changes more dramatically under different pillar heights. It must be noted that, the surface tension force between the top and bottom walls of the microchannel is a function of pillar height. With increase in pillar height, the surface area increases and enhances the flow of fluid in the channel. This increase is more obvious when the pillar height is doubled and the change is slow with further increase in the pillar height. This may be due to the effect of irreversible viscous dissipation resulting from a larger surface area.

Figure 7 shows the variation of meniscus centerline displacement with square root of time. It is observed that, there is linear variation of meniscus centerline displacement with square root of time depicting Lucas-Washburn behavior (Washburn 1921).

Figure 8 shows the meniscus centerline displacement with respect to time for different pillar pitch to diameter ratio $(p / d)$. The pitch is fixed at $650 \mu \mathrm{m}$ while the diameter of the pillar considered are 250,350 and $450 \mu \mathrm{m}$,

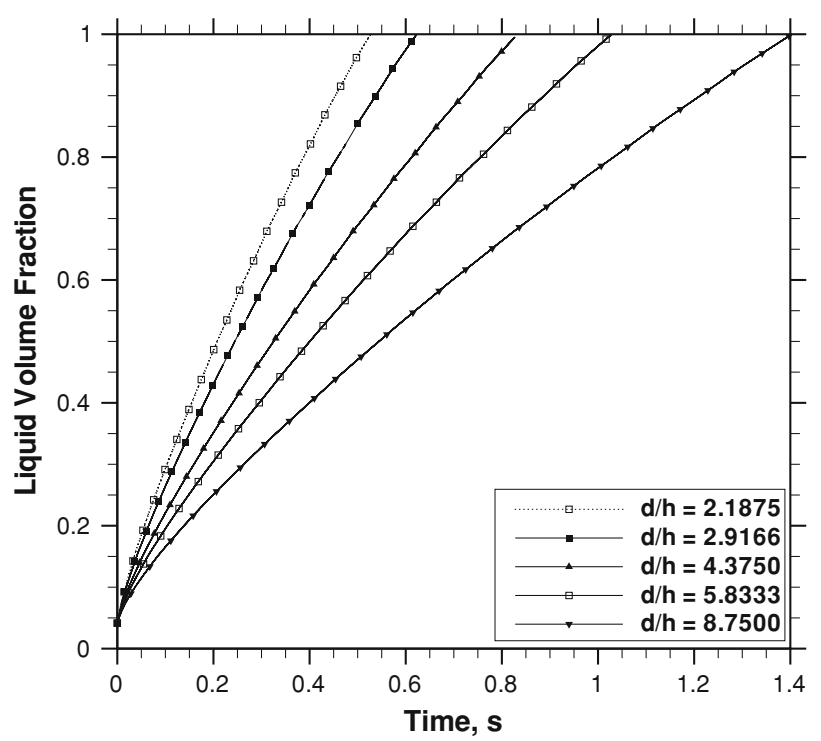

Fig. 6 Transient liquid phase volume fraction for different pillar diameter to height ratio with isopropyl alcohol 


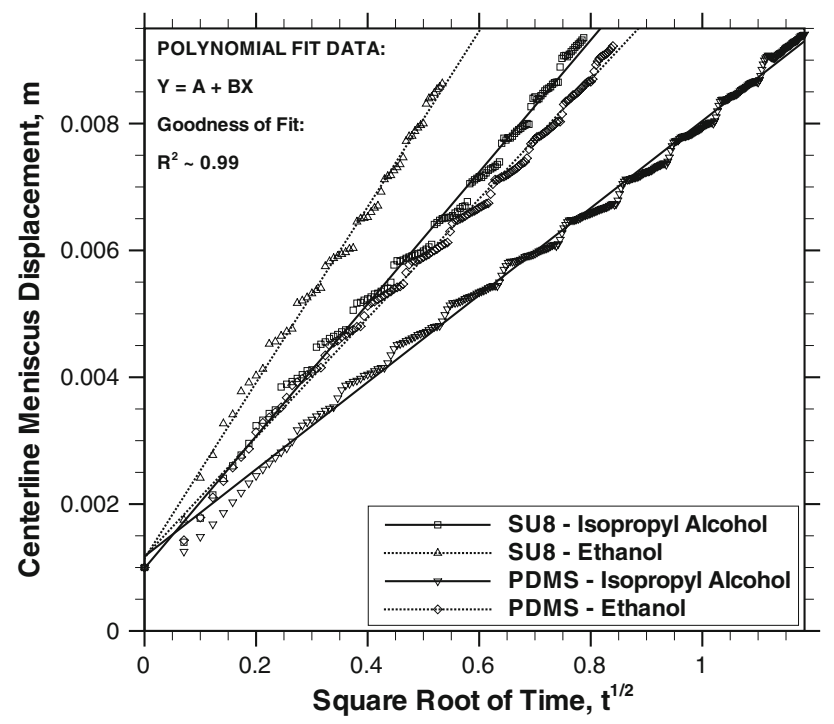

Fig. 7 Plot showing the liner variation of meniscus centerline displacement with square root of time for SU8 and PDMS channels using isopropyl alcohol and ethanol

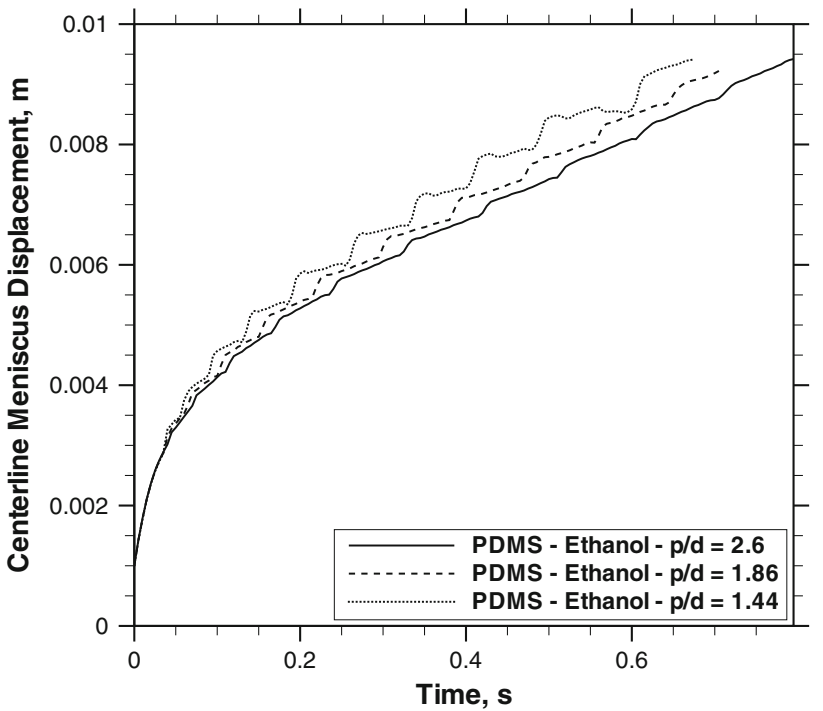

Fig. 8 Transient meniscus centerline displacement with time for microchannel with different pitch to diameter ratio

respectively. It is observed that, the capillary meniscus displacement is not uniform due to the presence of pillars. The flow is faster at the converging side of the gap and slows down at the diverging side and then becomes uniform where there are no pillars. The non-uniformity in meniscus displacement due to the presence of pillars is observed to increase with decrease in pitch to diameter ratio. It is also observed that a higher meniscus displacement is obtained with decrease in pitch to diameter ratio with a corresponding decrease in the liquid volume filling the microchannel. Figure 9 shows the variation of liquid volume filled with time.

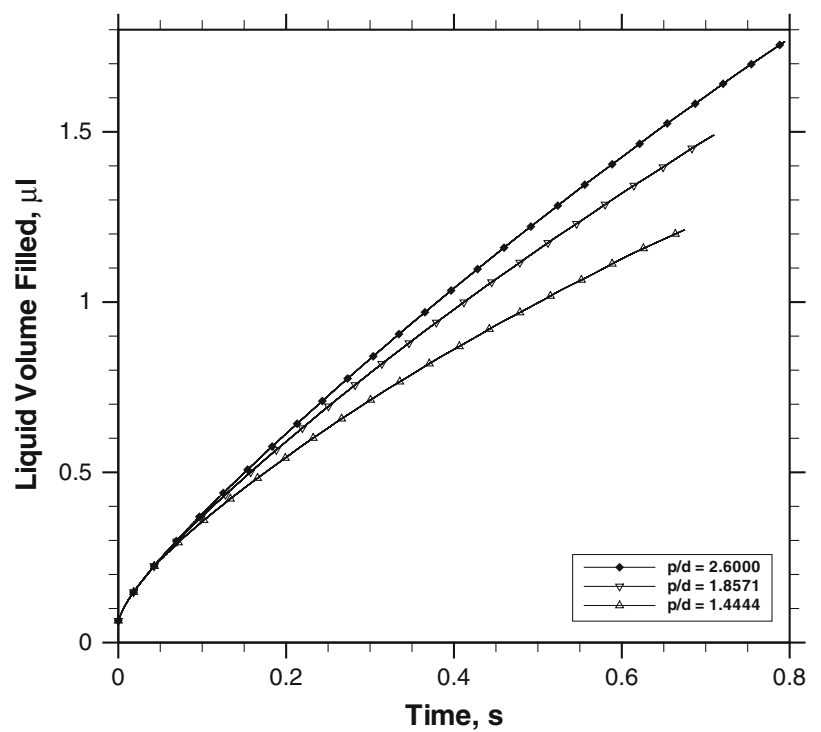

Fig. 9 Transient liquid volume of ethanol in PDMS microchannel at $d / h=8.75$ with different pitch to diameter ratio

To understand the effect of surface area to volume ratio on the capillary performance of the microchannel, the variation of meniscus centerline average velocity with different microchannel surface area to volume ratio is shown in Fig. 10. It is observed that with the same number of pillars $(n=88)$, by decreasing the pitch to diameter ratio the meniscus average centerline velocity increases. The increase in the velocity becomes more prominent with decreasing diameter to height ratios. Capillary forces are developed between the small gap between the pillars and the effect becomes predominant with decrease in pitch to diameter ratio.

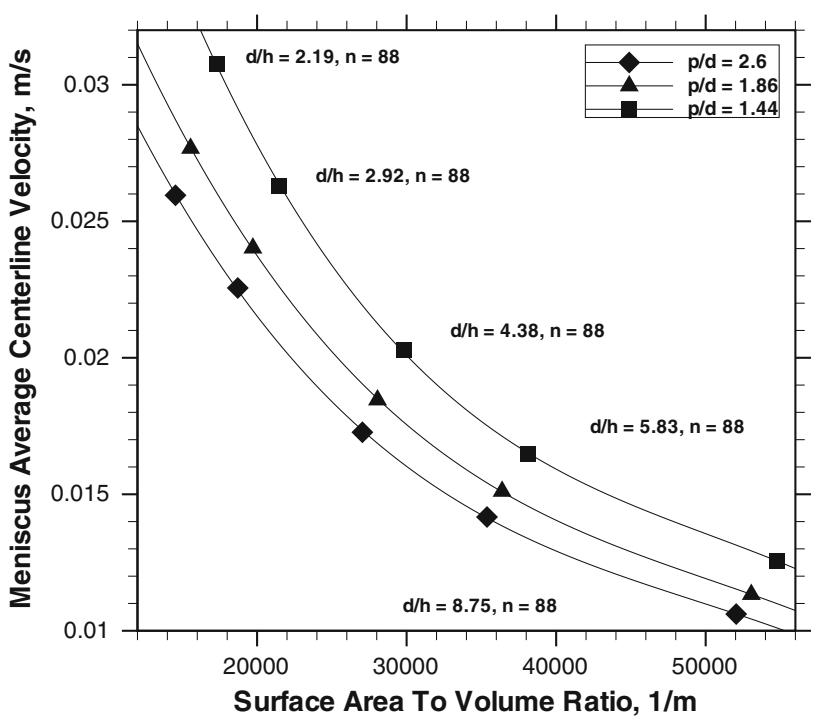

Fig. 10 Plot showing the variation of meniscus centerline average velocity of isopropyl alcohol with different microchannel surface area to volume ratio 
Figure 11 show the effect of viscosity and surface tension of the working medium on the capillary phenomena. It is observed that the capillary flow in the microchannel is very sensitive to the physical properties of the working medium and affects the simulated filling times. The effect of viscosity change is observed to be linear. Whereas, the effect becomes non-linear with change in surface tension of the fluid, as shown in Fig. 11b.

Figure 12 shows the the variation of liquid phase volume fraction with capillary number and dimensionless meniscus centerline displacement for different aspect ratio (pillar diameter to height ratio, $d / h$ ). The Capillary number (Ca), is defined as $\mu U / \sigma$, where $U$ is meniscus centerline

(a) Viscosity

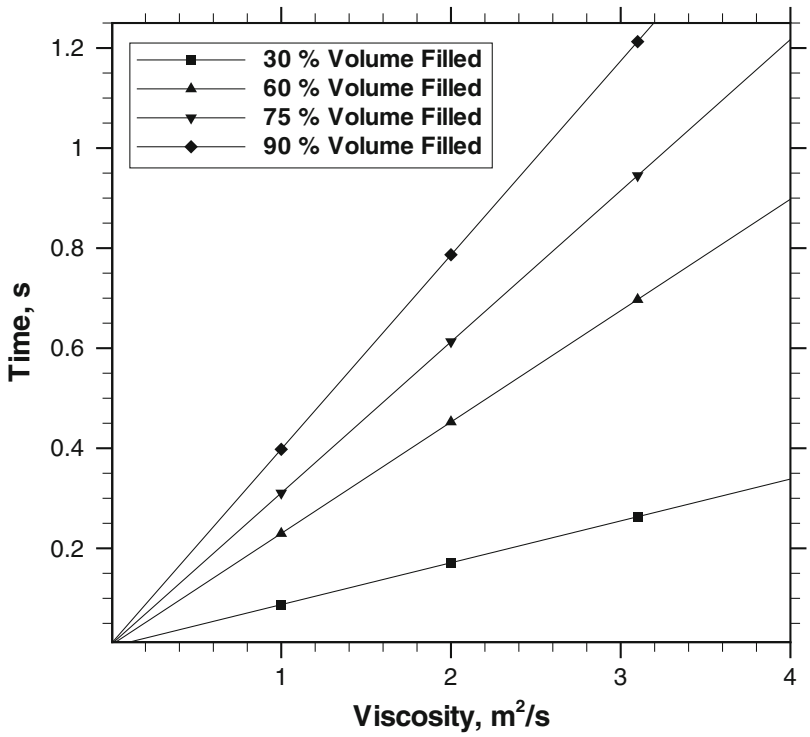

(b) Surface Tension

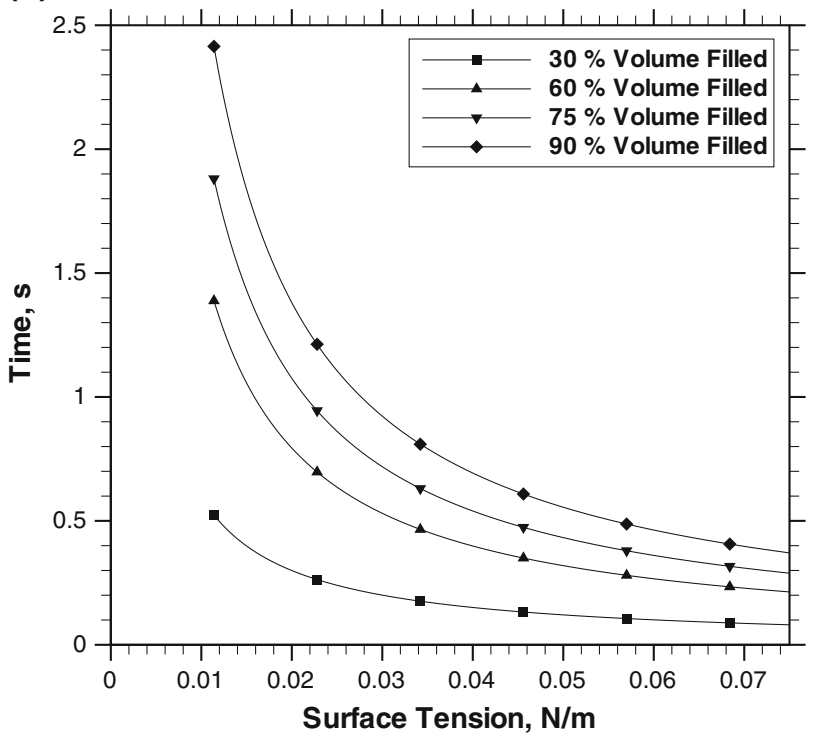

Fig. 11 Transient liquid phase volume fraction for different fluid viscosity and surface tension with $d / h=8.75$ velocity, $\mu$ and $\sigma$ are the fluid viscosity and surface tension, respectively. The flow is observed to have a very low capillary number, which signifies the relative importance of surface tension to viscous effects in the present study. As shown in Fig. 12a, the liquid phase volume fraction increases with decrease in capillary number, for a given aspect ratio. Since the meniscus centerline velocity decreases with increasing time, the Capillary number also decreases in accordance with the above $(\mathrm{Ca})$ equation. Figure $12 \mathrm{~b}$ shows the variation of dimensionless meniscus displacement (defined as ratio of meniscus centerline displacement to the total microchannel length of 9,450 $\mu \mathrm{m}$ ) with liquid phase volume fraction for different aspect ratio
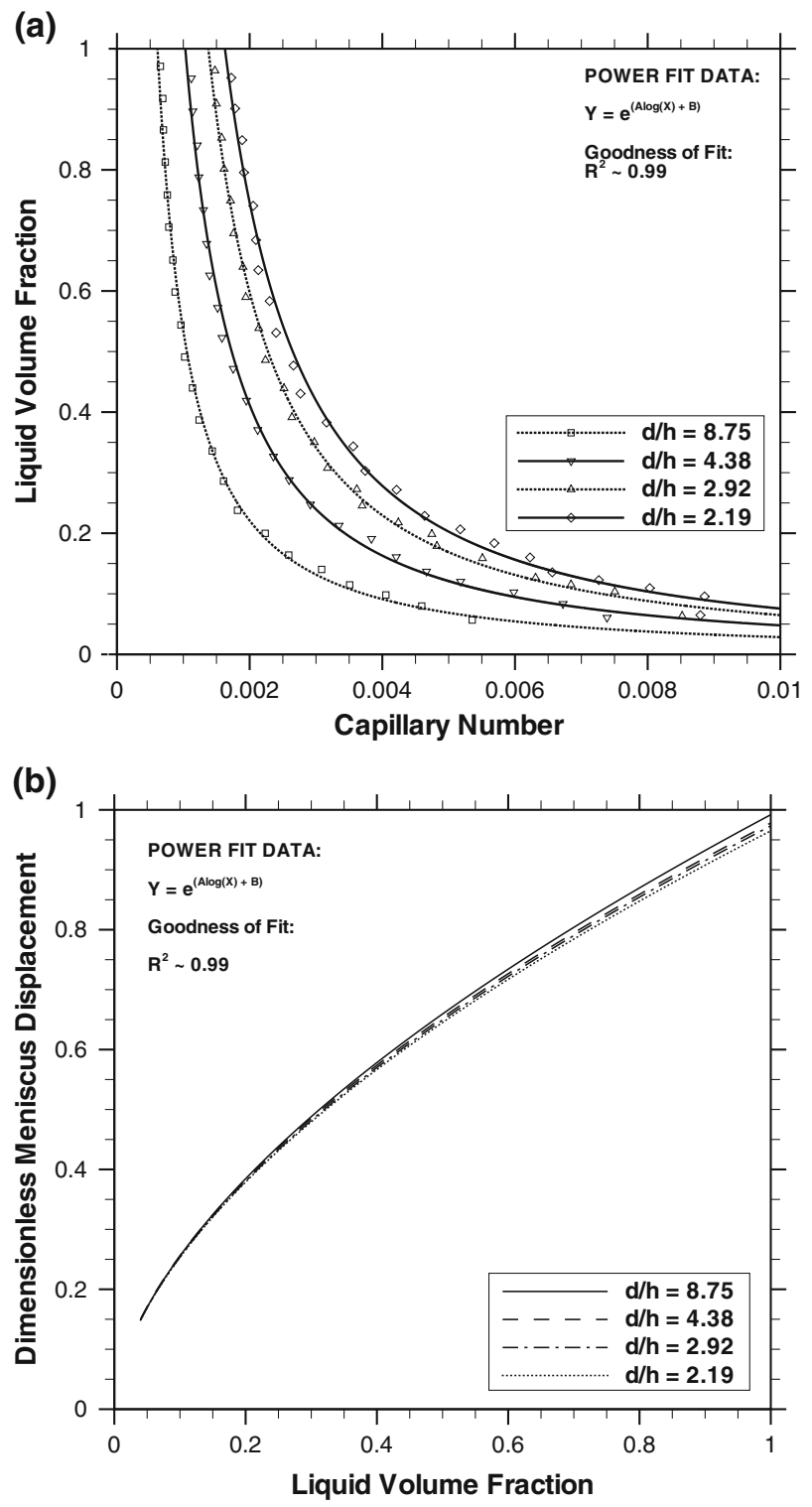

Fig. 12 Variation of liquid phase volume fraction for different aspect ratio (pillar diameter to height ratio $d / h$ ). a Capillary number, b dimensionless meniscus displacement 
(pillar diameter to height ratio, $d / h$ ). It is observed that, there is a non linear variation of dimensionless meniscus displacement with liquid phase volume fraction. This relates the meniscus centerline displacement with the volume of fluid filled for different aspect ratios. It is observed that with decrease in aspect ratio a lower liquid phase volume fraction is obtained for a fixed dimensionless meniscus displacement. This is more prominent at higher values of dimensionless meniscus centerline displacement. The lower liquid phase volume fraction may be due to the irreversible viscous dissipation resulting from a larger surface area when aspect ratio is less. These results can be used for quick estimates of the performance parameters for the design of the microfluidic devices.

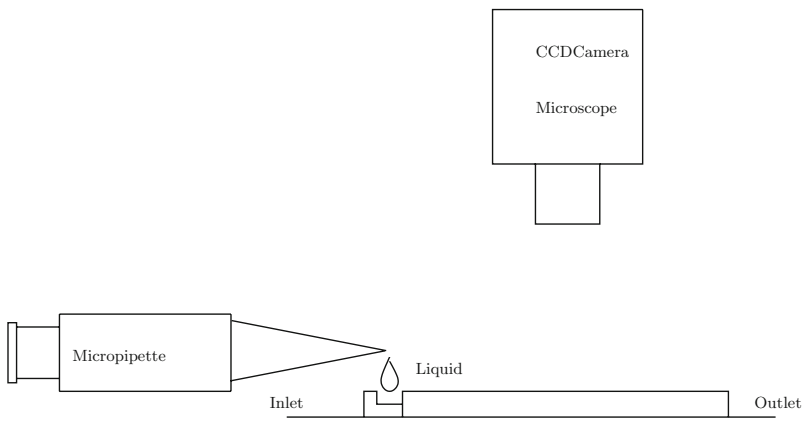

Fig. 13 Schematic drawing of experimental setup

\subsection{Experimental verification}

Droplets of ethanol and isopropyl alcohol are introduced in the microchannel reservoir using a micro pipette $(0.5-$ $10 \mu \mathrm{l})$ so that adequate volume of droplets are dispensed for effective passive capillary flow. Figure 13 shows the schematic drawing of experimental setup. Many successive runs were performed for each of the test fluids. After each experiment, the liquid in the microchannel is blown away to set the initial condition in the channel free of any liquid traces. We have allowed sufficient time to ensure no trace of liquid is left before the commencement of repeat runs. From the successive runs of isopropyl alcohol and ethanol, the interface motion seems to be well repeatable under identical conditions. It was also observed during the experiments that, always a thin liquid film is formed adjacent to the meniscus interface for both the working medium. Thus the interface motion is always wet due to the high wetting properties of the test liquids for glass, PDMS and SU8 surfaces.

\subsubsection{SU8 microchannel}

The snapshot image of the time evolution of meniscus front is shown in Fig. 14 for isopropyl alcohol. The red color indicates the liquid volume fraction $(F=1)$ as the fluid

Fig. 14 The snapshot image of the time evolution of meniscus front of isopropyl alcohol in microchannel (Numerical)

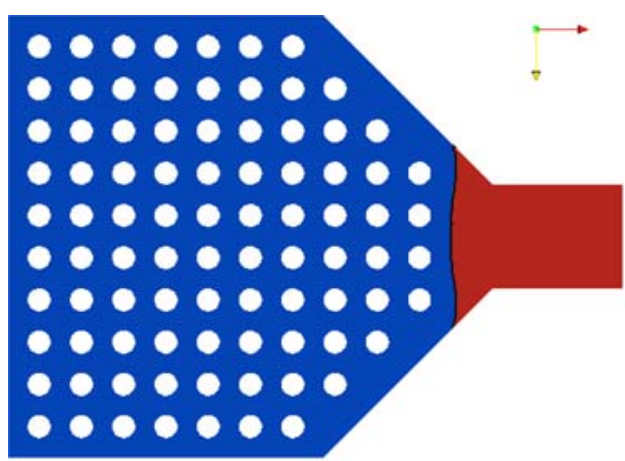

(a) $\mathrm{t}=0.025 \mathrm{~s}$

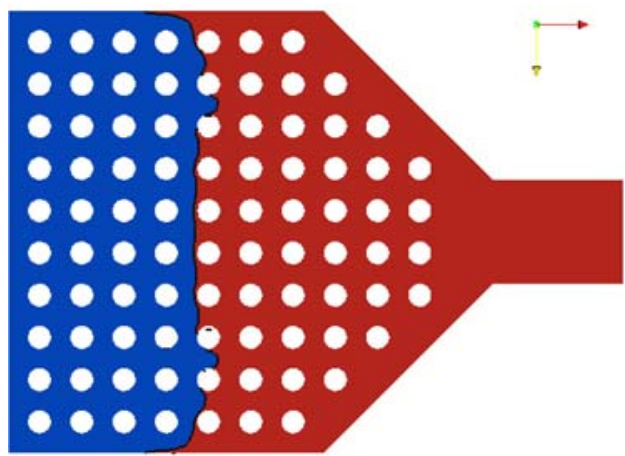

(c) $\mathrm{t}=0.300 \mathrm{~s}$

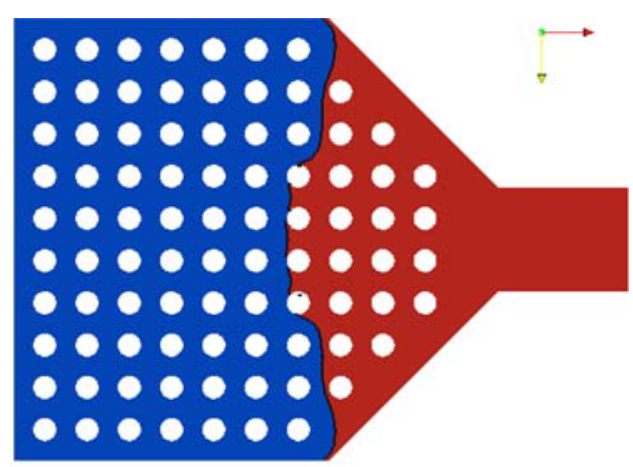

(b) $\mathrm{t}=0.150 \mathrm{~s}$

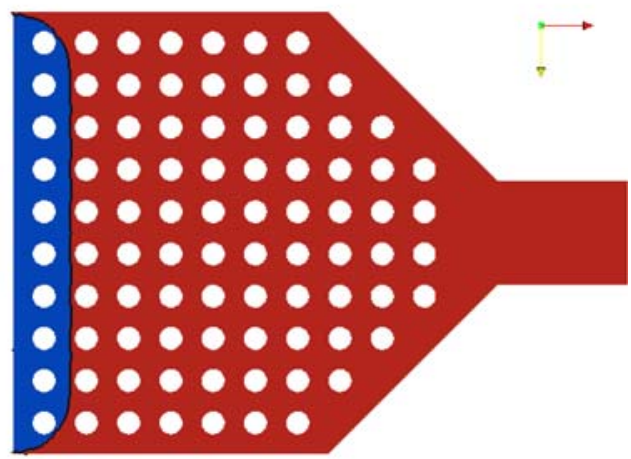

(d) $\mathrm{t}=0.515 \mathrm{~s}$ 
advances in the microchannel while blue color indicates air $(F=0)$. The corresponding experimental snapshot image of the time evolution of meniscus front is shown in Fig. 15 obtained using the inverted microscope.

Interesting observations can be drawn from the meniscus profiles obtained at the microchannel neck (Figs. 14a, 15a), diverging portion (Figs. 14b, 15b) and straight length section (Figs. 14c, 15c) of the channel for isopropyl alcohol. The surface inside the microchannel is hydrophilic as the walls are considered to be fully wet. The meniscus undergoes topological transformations and evolves between the pillar structure with concave profile. A concave profile of the meniscus is observed in the neck and diverging section of the microchannel due to the hydrophilic side walls. As the meniscus crosses the diverging section, the meniscus attains uniform profile due to the presence of pillars in the straight length section. The evolution of the meniscus between the pillars is also uniform. It is interesting to note that, the meniscus displacement along the microchannel side wall is slightly faster compared to centerline meniscus displacement. Experimental observations have also shown the above phenomena. The simulated meniscus profiles show good agreement with the experimental images for isopropyl alcohol and the presence of pillars creates uniform meniscus front in the microchannel.

\subsubsection{PDMS microchannel}

Figure 16 shows the comparison of simulation and stereomicroscope images of the time evolution of meniscus front for PDMS microchannel with isopropyl alcohol and ethanol as working fluid, respectively. The simulated meniscus profiles show good agreement with the experimental
Fig. 15 The snapshot image of the time evolution of meniscus front of isopropyl alcohol in microchannel (Experiment)

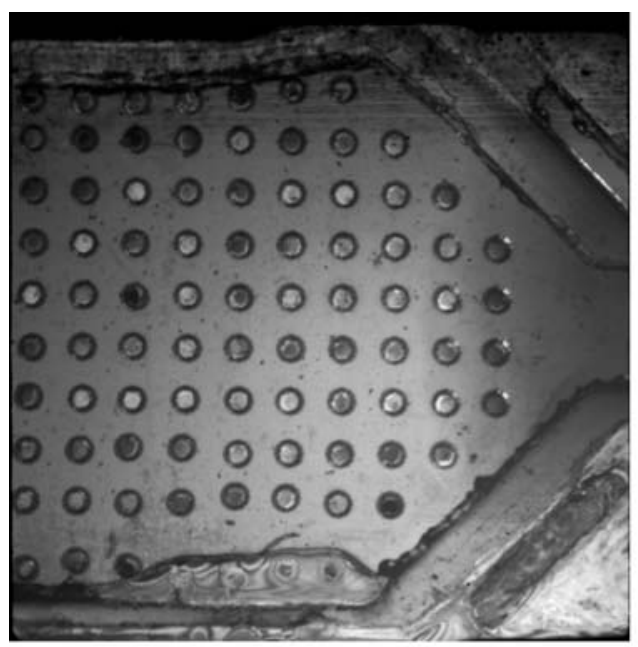

(a) $\mathrm{t}=0.091 \mathrm{~s}$

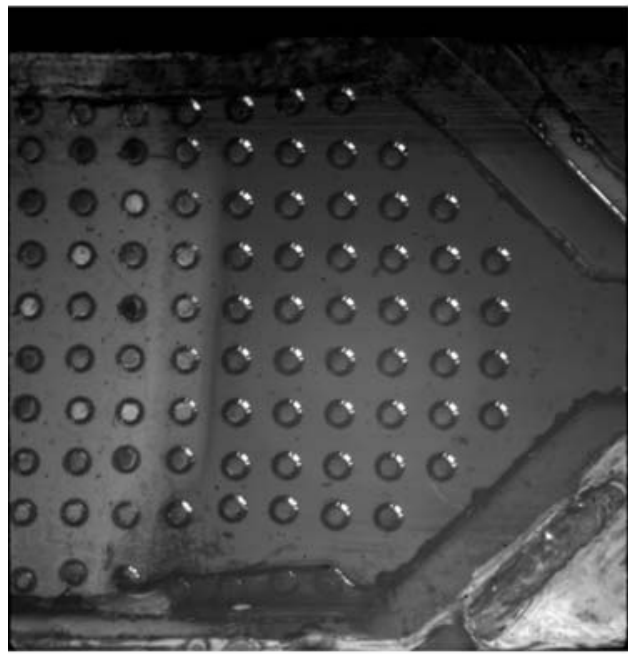

(c) $\mathrm{t}=0.453 \mathrm{~s}$

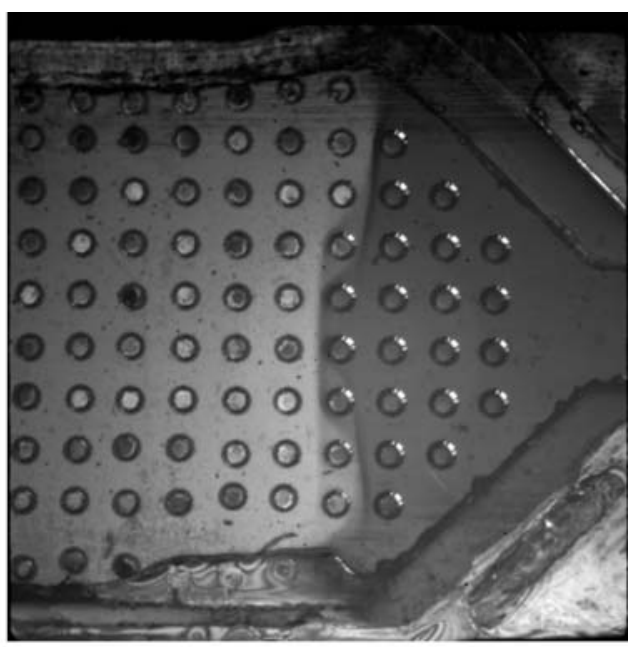

(b) $\mathrm{t}=0.188 \mathrm{~s}$

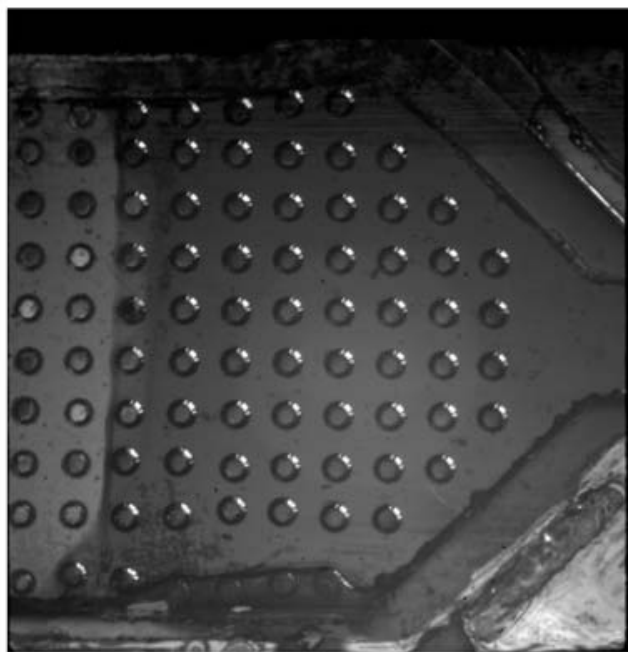

(d) $\mathrm{t}=0.609 \mathrm{~s}$ 
Fig. 16 The snapshot image of the time evolution of meniscus front in PDMS microchannel (Experiment and Numerical)
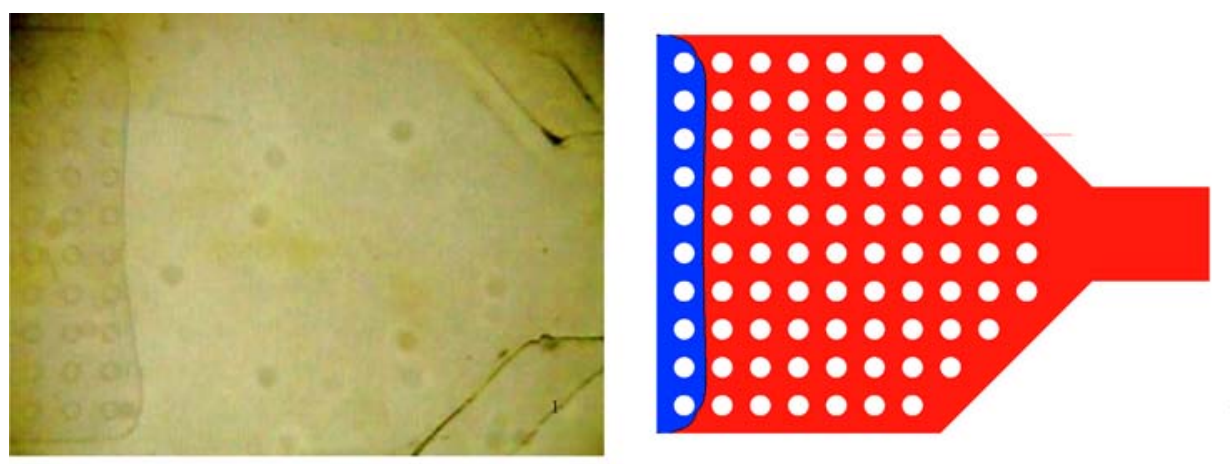

(a) Isopropyl Alcohol. (1) Experiment - Time $=1.26 \mathrm{~s}(2)$ Numerical - Time $=1.185 \mathrm{~s}$
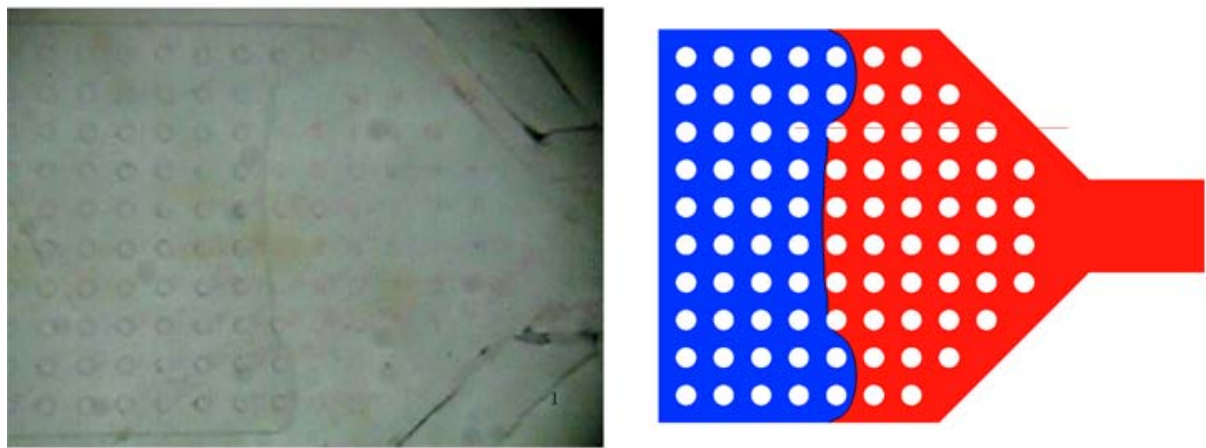

(b) Ethanol. (1) Experiment - Time $=0.33 \mathrm{~s}$ (2) Numerical - Time $=0.345 \mathrm{~s}$

images for both isopropyl alcohol and ethanol and the presence of pillars creates uniform meniscus front in the microchannel.

Figure 17 shows the numerical and experimental comparison of meniscus centerline velocity obtained with isopropyl alcohol for SU8 and PDMS microchannel. It is observed that, the meniscus centerline velocity obtained experimentally $(22,919 \mu \mathrm{m} / \mathrm{s})$ is slightly lower compared to the simulated $(23,890 \mu \mathrm{m} / \mathrm{s})$ value for SU8 microchannel. This may be due to improper sealing between the glass slide and the substrate. This may create a small air gap between the sealing surfaces which could provide resistance to the capillary flow. It was also observed that the dispensed droplet volume at the reservoir effects the capillary flow in the SU8 devices due to its higher internal volume. A smaller volume of liquid at the reservoir inlet of SU8 microchannel tends to have a slower meniscus displacement as it is starved for adequate volume of liquid to fill the complete channel length. The effect of dispensed droplet volume at the reservoir for PDMS device was not critical due to its lower internal volume. The movement of the meniscus is observed to be no longer symmetric about the channel width under such circumstances. However, for the PDMS microchannel, the average meniscus centerline velocity obtained experimentally $(13,386 \mu \mathrm{m} / \mathrm{s})$ is slightly higher compared to the simulated value $(11,280 \mu \mathrm{m} / \mathrm{s})$. The observed discrepancy may be attributed to the geometrical inaccuracies inherent to the fabrication of the PDMS device. Other possible reason of discrepancy the authors presume, may be due to the adsorption of working fluid on the PDMS bottom wall.

Figure 18 shows the summary comparison of the meniscus displacement measured experimentally plotted with respect to those obtained from the numerical solution for all the geometries and fluids considered. The diagonal line in the plot corresponds to the numerical solution and the dashed lines denote the $10 \%$ error bands. Of the total number of 43 data points in the figure, $85.00 \%$ are within $\pm 10 \%$ error bands, which demonstrates the validation of the current numerical analysis for all the geometries and fluids considered. Thermophysical properties are corrected with temperature measured before experiments.

It must be noted that, it is difficult to precisely replicate the experimental conditions in simulation, particularly for capillary driven flow. Initially, at time $t=0$, the liquid meniscus position in the channel is set as $1.00 \mathrm{~mm}$ in the simulation. We have observed that, maintaining this initial length of liquid front during experiments is a very difficult task. Other limitations which prohibit the accurate determination of the transient meniscus evolution are the field of view of the microscope to accommodate the entire length and width of the microchannel and the image capturing capability of the CCD camera. 


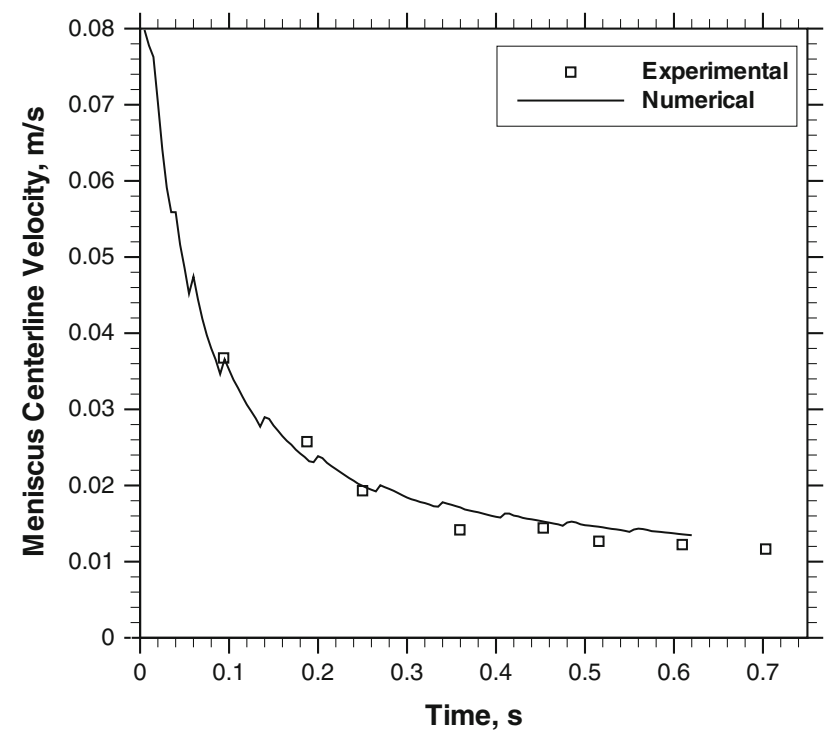

(a) $\mathrm{SU} 8$

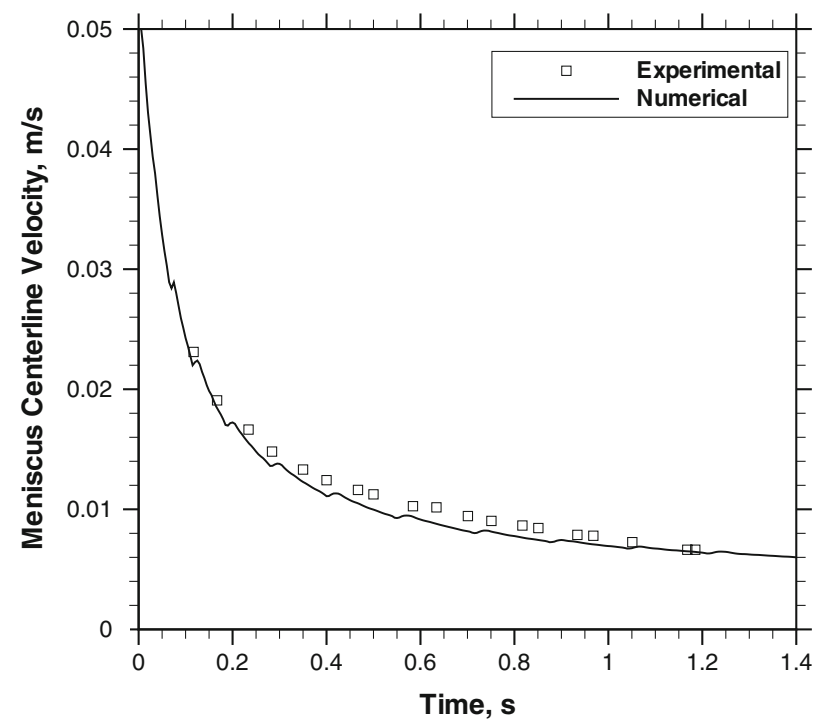

(b) PDMS

Fig. 17 Numerical and experimental comparison of meniscus centerline velocity obtained with isopropyl alcohol

There are some fabrication related issues to sealing the SU8 and PDMS channels. The channels are normally sealed with glass slides. A good reversible sealing was obtained with PDMS, whereas, the sealing for SU8 offered few problems during the fabrication. However, the fabrication of SU8 microchannels was fairly simple with few steps. SU8 is going to be the preferred material for high performance microfluidic devices, if the sealing issues are addressed in these devices.

Taking the above limitations into account, the obtained experimental results are fairly accurate and agree well with the computational simulations.

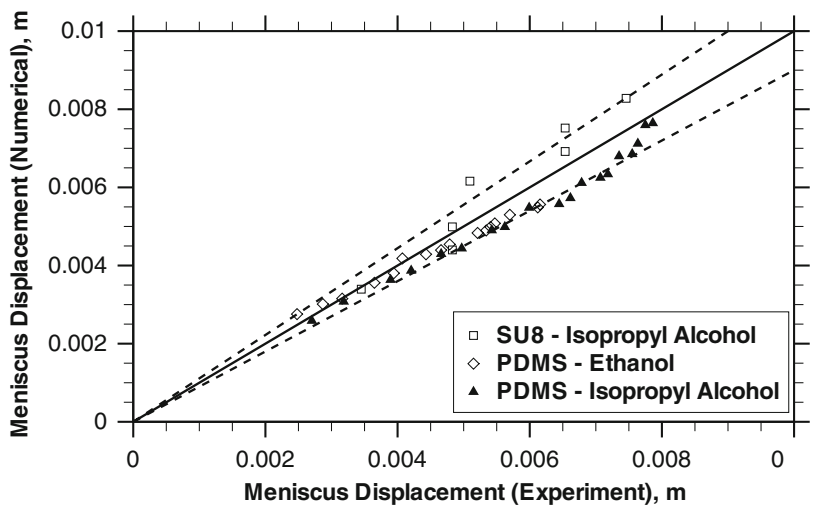

Fig. 18 Summary comparison of numerical solution with experimental results on the meniscus displacement for all the geometries and fluids considered

\section{Conclusions}

Three-dimensional numerical simulation of passive capillary flow of ethanol and isopropyl alcohol in microfluidic channels with integrated pillars is presented here. The simulations were carried out for geometries based on SU8 and PDMS substrates with different pillar diameter to height ratio, ranging from 2.19 to 8.75 and pillar diameter to pitch ratio, ranging from 1.44 to 2.6. The geometry of the pillar and the physical properties of the fluid are found to have significant influence on the capillary phenomena in the microchannel. A higher velocity of the capillary meniscus is obtained with increase in surface area to volume ratio in the devices. Also, generalized plots have been obtained in terms of dimensionless variables to predict the liquid volume fraction in the microchannel. With decrease in pillar diameter to height ratio, a lower liquid phase volume fraction is obtained for a fixed dimensionless meniscus displacement. The transient evolution of meniscus front is experimentally visualized in SU8 and PDMS channel using stereo and inverted microscope for both ethanol and isopropyl alcohol, respectively. Numerical predictions agree well with the experimental observations for both SU8 and PDMS microchannels. The simulated meniscus profiles show good agreement with the experimental images for both the working medium considered in the study. It is observed that the filling time obtained experimentally matches very well with the simulated filling time. The numerical results provided good qualitative as well as quantitative insight into the capillary phenomena in the microchannel. The presence of pillars creates uniform meniscus front in the microchannel for both ethanol and isopropyl alcohol. The study will help in designing high performance microfluidic devices for performing microfluidic analysis in the presence of pillars. 
Acknowledgments The support of Suman Mashruwala Advanced Microengineering Laboratory, IIT Bombay is highly appreciated. The authors also like to acknowledge the UKIERI funding provided to $\mathrm{S}$. K. Mitra and J. McLaughlin to carry out this collaborative work.

\section{References}

Barraza HJ, Kunapuli S, O’Rear EA (2002) Advancing contact angles of Newtonian fluids during high velocity, transient, capillary driven flow in a parallel plate geometry. J Phys Chem B 106:4979-4987

Bayramli E, Powell RL (1989) The normal (transverse) impregnation of liquids into axially oriented fibre bundles. J Colloid Interface Sci 138(2):346-353

Brackbill JU, Kothe DB, Zemach C (1992) A continuum method for modeling surface tension. J Comp Phys 100:335-354

Chakraborty S (2005) Dynamics of capillary flow of blood into microfluidic channel. Lab Chip 5:421-430

Chakraborty S (2007a) Order parameter modeling of fluid dynamics of narrow confinements subjected to hydrophobic interactions. Phys Rev Lett 99:094504

Chakraborty S (2007b) Towards a generalized representation of surface effects on pressure-driven liquid flow in microchannels. Appl Phys Lett 90:034108

Chakraborty S (2007c) Electroosmotically driven capillary transport of typical non-Newtonian biofluids in rectangular microchannels. Anal Chim Acta 605:175-184

Chakraborty S, Mittal R (2007) Droplet dynamics in a microchannel subjected to electrocapillary actuation. J Appl Phys 101:104901

Chakraborty S, Das T, Chattoraj S (2007) A generalized model for probing frictional characteristics of pressure-driven liquid microflows. J Appl Phys 102:104907

Chakraborty S, Tsuchiya K (2008) Development and fluidic simulation of microneedles for painless pathological interfacing with living systems. J Appl Phys 103:114701

Chakraborty S, Dinkar AK (2008) Implications of hydrophobic interactions and consequent apparent slip phenomenon on the entrance region transport of liquid through microchannels. Phys Fluids 20:043602

Chen YF, Tseng FG, Chien SYC, Chen MH, Yu RJ, Chieng CC (2008) Surface tension driven flow for open microchannels with different turning angles. Microfluid Nanofluid 5(2):193-203

Hirt CW, Nichols BD (1981) Volume of fluid (VOF) method for the dynamics of free boundaries. J Comp Phys 39:201-225

Huang W, Liu Q, Li Y (2006) Capillary filling flows inside patternedsurface microchannels. Chem Eng Technol 26:716-723

Jensen MJ (2002) Bubbles in microchannels. MSc thesis, Technical University of Denmark, Denmark

Jimack PK (2004) Adaptive algorithms for free-surface flow problems. In: Proceedings of the 4th international conference on engineering computational technology, Lisbon, Portugal, pp 1-24

Jokinen V, Franssila S (2008) Capillarity in microfluidic channels with hydrophilic and hydrophobic walls. Microfluid Nanofluid 5:443-448

Kern P, Veh J, Michler J (2007) New developments in through-mask electrochemical micromachining of titanium. J Micromech Microeng 17:1168-1177

Kim B, Peterson ETK, Papautsky I (2004) Long-term stability of plasma oxidized PDMS surfaces. In: Proceedings of the 26th annual international conference of the IEEE EMBS, San Francisco, pp 5013-5016
Lin CM (2004) Enhancement of underfill capillary flow in flip-chip packaging by means of the inertia effect. IEEE Trans Adv Packag 27(3):533-539

Lin CM, Chang WJ, Fang TH (2007) Flip-Chip underfill packaging considering capillary force, pressure difference, and inertia effects. J Electron Packag 129:48-55

Losey MW, Jackman RJ, Firebaugh SL, Schmidt MA, Jensen KF (2002) Design and fabrication of microfluidic devices for multiphase mixing and reaction. J Microelectromech Syst 11(6):709-717

McDonald JC, Whitesides GM (2002) Poly(dimethylsiloxane) as a material for fabricating microfludic devices. Acc Chem Res 35(7):491-499

Mery E, Ricoul F, Sarrut N, Constantin O, Delapierre D, Garin J, Vinet F (2008) A silicon microfluidic chip integrating an ordered micropillar array separation column and a nano-electrospray emitter for LC/MS analysis of peptides. Sens Actuators B 134:438-446

Nissila T, Sainiemi L, Sikanen T, Kotiaho T, Franssila S, Kostiainen R, Ketola RA (2007) Silicon micropillar array electrospray chip for drug and biomolecule analysis. Rapid Commun Mass Spectrom 21:3677-3682

OpenCFD Ltd (2008) OpenFOAM user guide. Version 1.5 ed

Probstein R (1994) Physicochemical hydrodynamics. Wiley, New York

Quinte A, Halstenberg S, Eggert H (2001) Use of numerical methods for modeling and simulating capillary driven flows in microchannels. Nanotech 2001:250-253

Rusche H (2002) Computational fluid dynamics of dispersed twophase flows at high phase fractions. $\mathrm{PhD}$ thesis, Imperial College, University of London, London

Seemann R, Kramer EJ, Lange FF (2004) Patterning of polymers: precise channel stamping by optimizing wetting properties. New J Phys 6:110

Sethian JA, Smereka P (2003) Level set methods for fluid interfaces. Ann Rev Fluid Mech 35:341-372

Son SU, Seo JH, Choi YH, Lee SS (2006) Fabrication of a disposable biochip for measuring percent hemoglobin Alc (\% HbAlc). Sens Actuators A 130-131:267-272

Thorslund S, Larsson R, Bergquist J, Nikolajeff F, Sanchez J (2008) A PDMS-based disposable microfluidic sensor for CD4+ lymphocyte counting. Biomed Microdevices 10:851-857

Tseng FG, Yang ID, Lin KH, Ma KT, Lu MC, Tseng YT, Chieng CC (2002) Fluid filling in micro-fabricated reservoirs. Sens Actuators A 97-98:131-138

Ubbink O, Issa RI (1999) A method for capturing sharp fluid interfaces on arbitrary meshes. J Comp Phys 153:26-50

Wan JW, Zhang WJ, Bergstrom DJ (2005) Influence of transient flow and solder bump resistance on underfill process. Microelectron $\mathrm{J}$ 36(8):687-693

Wan JW, Zhang WJ, Bergstrom DJ (2007) Recent advances in modeling the underfill process in flip-chip packaging. Microelectron J 38:67-75

Washburn EW (1921) The dynamics of capillary flow. Phys Rev 17:273-299

Xiao Y, Yang F, Pitchumani R (2006) A generalized analysis of capillary flows in channels. J Colloid Interface Sci 298:880-888

Zeng J (2007) On modeling of capillary filling. http://www.coventor. com/pdfs/on_modeling_of_capillary_filling.pdf. Accessed on 1503-2007

Zimmermann M, Bentley S, Schmid H, Hunziker P, Delamarche E (2005) Continuous flow in open microfluidics using controlled evaporation. Lab Chip 5:1355-1359 